\title{
Quantifying the Transmit Diversity Order of Euclidean Distance Based Antenna Selection in Spatial Modulation
}

\author{
Rakshith Rajashekar, Member, IEEE, K. V. S. Hari, Fellow, IEEE, and L. Hanzo, Fellow, IEEE
}

\begin{abstract}
In this letter, we quantify the transmit diversity order of the SM system operating in a closed-loop scenario. Specifically, the SM system relying on Euclidean distance based antenna subset selection (EDAS) is considered and the achievable diversity gain is evaluated. Furthermore, the resultant trade-off between the achievable diversity gain and switching gain is studied. Simulation results confirm our theoretical results. Specifically, at a symbol error rate of about $10^{-4}$ the signal-to-noise ratio gain achieved by EDAS is about $7 \mathrm{~dB}$ in case of 16-QAM and about $5 \mathrm{~dB}$ in case of 64-QAM.
\end{abstract}

Index Terms-Antenna selection, diversity, limited feedback, spatial modulation, switching gain.

\section{INTRODUCTION}

$\mathbf{C}$ ONSIDER a spatial modulation (SM) based system [1]-[9] having $N_{t}$ transmit antennas (TA) and $N_{r}$ receive antennas (RA), whose system model is given by

$$
\mathbf{y}=\sqrt{\rho} \mathbf{h}_{i} s+\mathbf{n},
$$

where $\rho$ is the average signal-to-noise ratio (SNR) at each receive antenna, $\mathbf{y} \in \mathbb{C}^{N_{r}}$ is the received signal vector, $\mathbf{h}_{i}$ is the channel vector corresponding to the $i$ th TA, $s$ is a random symbol selected from a unit-energy $M$-QAM or $M$-PSK signal set represented by $S$ and $\mathbf{n} \in \mathbb{C}^{N_{r}}$ is the noise vector. The entries of both channel matrix $\mathbf{H}$ and of the noise vector $\mathbf{n}$ are from a circularly symmetric complex-valued Gaussian distribution $\mathcal{C N}(0,1)$. The input bitstream in SM is divided into blocks of $\log _{2}\left(N_{t} M\right)$ bits and in each such block, $\log _{2} M$ bits select a symbol $s$ from an $M$-QAM or $M$-PSK signal set, while $\log _{2} N_{t}$ bits per channel use (bpcu) select an antenna $i$ out of $N_{t}$ transmit antennas for the transmission of the selected symbol $s$. Thus, the SM system achieves $\log _{2} N_{t}$ bits higher throughput than the

Manuscript received November 06, 2014; revised February 16, 2015; accepted February 25, 2015. This work was supported by the DST, India, and by the EPSRC, U.K., under the auspices of the India-UK Advanced Technology Center (IU-ATC). The associate editor coordinating the review of this manuscript and approving it for publication was Prof. Zoltan Safar.

R. Rajashekar and K. V. S. Hari are with the Department of Electrical Communication Engineering, Indian Institute of Science, India (e-mail: rakshithmr@ece.iisc.ernet.in; hari@ece.iisc.ernet.in).

L. Hanzo is with the School of ECS, University of Southampton, UK (e-mail: 1h@ecs.soton.ac.uk).

Color versions of one or more of the figures in this paper are available online at http://ieeexplore.ieee.org.

Digital Object Identifier 10.1109/LSP.2015.2408574 single-input multiple-output (SIMO) system using $M$-ary modulation.

Definition 1: In the SM system, the number of bits transmitted per channel use through the TA indices is defined as the spatial switching gain (SSG) of the SM system.

Although SM has the benefit of having a single RF chain, the related disadvantage is that it has no transmit diversity gain due to the activation of single TA in every channel use. Some recently proposed schemes to increase the diversity order of SM beyond one are time-orthogonal signal design assisted spatial modulation [3], [10], coherent space-time shift keying [11], time-orthogonal signal design assisted SM relying on STBC [12] and space-time block coded spatial modulation [13]. These schemes constitute some of the existing open-loop techniques conceived for SM. Recently, some closed-loop techniques were conceived for the SM system [14]-[16], where the SM transmitter relies on the feedback information sent back from the receiver. In this paper, we focus our attention on the Euclidean distance (ED) based antenna subset selection (EDAS) technique [14]-[16], which is briefly described as follows.

$\boldsymbol{E D A S}$ : Let $N_{S M}$ out of $N_{t}$ TAs be selected for achieving a SSG of $\log _{2} N_{S M}$ bpcu. Let $\mathcal{I}=\left\{I_{j}\right\}_{j=1}^{n}$ represent the set of enumerations of all possible $n=\left(\begin{array}{c}N_{t} \\ N_{S M}\end{array}\right)$ combinations of selecting $N_{S M}$ out of $N_{t}$ TAs. Among the $\left(\begin{array}{c}N_{t} \\ N_{S M}\end{array}\right)$ possibilities, the specific TA set that maximizes the minimum ED among all possible transmit vectors (TV) [15] is obtained as

$$
I_{E D}=\arg \max _{I \in \mathcal{I}}\left\{\min _{\mathbf{x}_{1} \neq \mathbf{x}_{2} \in \chi}\left\|\mathbf{H}_{I}\left(\mathbf{x}_{1}-\mathbf{x}_{2}\right)\right\|_{2}^{2}\right\},
$$

where $\mathbf{H}_{I} \in \mathbb{C}^{N_{r} \times N_{S M}}$ has $N_{S M}$ columns given by $I$ and $\chi$ represents the set of all possible transmit vectors given by $\left\{\mathbf{e}_{i} s\right\}_{i=1}^{N_{S M}}$, where $s \in S$ and $\mathbf{e}_{i}$ is a $N_{S M} \times 1$ vector having 1 as the only non-zero element at the $i$ th location, with $|\chi|=$ $N_{S M} M$. The chosen $I_{E D}$ is encoded into bits, which are then sent to the transmitter once every coherence interval. Upon receiving this information, the transmitter starts data transmission through the TA indexed by the set $I_{E D}$. Note that the conventional TA selection scheme [17]-[19] is a special case of EDAS corresponding to $N_{S M}=1$.

Prior Work: A pair of TA selection schemes were proposed in [16], namely capacity optimized antenna selection (COAS) and EDAS. Furthermore, the components of the SM symbol error at the receiver were studied. Explicitly, it was shown that if $P_{e}(S M)$ represents the SM symbol error with components 
$\operatorname{Pr}(\mathcal{A})$ and $\operatorname{Pr}\left(S, \mathcal{A}^{c}\right)$, where $\mathcal{A}^{c}$ represents the event of an antenna index not being in error and $S$ represents the event of a transmitted symbol error under ML detection [16], then the component $\operatorname{Pr}\left(S, \mathcal{A}^{c}\right)$ in COAS achieves a diversity order of $N_{t}-N_{S M}+1, \log _{2} N_{S M}$. However, the effective diversity order of COAS was shown to be only $N_{r}$ owing to $\mathcal{A}$.

New Contributions: The EDAS of SM systems was shown to achieve significant symbol-error rate (SER) improvements over both COAS and over the conventional SM system. However, to the best of our knowledge, the diversity order of this system has not been quantified in the open literature. Hence in this paper, we show that SM employing EDAS will achieve a transmit diversity order of $d_{t}=\left(N_{t}-N_{S M}+1\right)$, and strikes an attractive trade-off between the transmit diversity and $S S G$, given by $\left(d_{t}, S S G\right)=\left(N_{t}-N_{S M}+1, \log _{2} N_{S M}\right)$. A further substantial benefit of antenna selection is that the number of high-cost radio-frequency chains is reduced.

Notations: The lowercase boldface letters represent vectors and uppercase boldface letters represent matrices. The notations of $\|\cdot\|$ and $\|\cdot\|_{F}$ represent the two-norm of a vector and the Frobenious norm of a matrix, respectively. Trace of a matrix is represented by $\operatorname{Tr}(\cdot)$. The notations of $(\cdot)^{T}$ and $(\cdot)^{H}$ indicate the transpose and Hermitian transpose of a vector/matrix, respectively, while $|\cdot|$ represents the cardinality of a given set, or the magnitude of a complex quantity. Expected value of a random variable $X$ is denoted by $\mathrm{E}(X)$, while the smallest non-zero Eigenvalue of matrix $\mathbf{Y}$ is denoted by $\lambda_{s}(\mathbf{Y})$. Furthermore, $Q(x)$ represents the tail probability of standard normal distribution given by $\frac{1}{\sqrt{2 \pi}} \int_{x}^{\infty} \exp \left(-\frac{u^{2}}{2}\right) d u$.

\section{MAIN RESUlts}

Let $L=\{i\}_{i=1}^{N_{t}}$ represent the set of TA indices and $\mathcal{C}_{i}=$ $\left\{s \mathbf{e}_{j} \mid s \in S, j \in I_{i}\right\}$ for $1 \leq i \leq n$ represent the $n$ possible subsets of TVs, where $\mathbf{e}_{j}$ is the $j^{\text {th }}$ column of the $\left(N_{t} \times N_{t}\right)$-element identity matrix. Let $\Delta \mathcal{C}_{i}=\left\{\mathbf{z}_{1}-\mathbf{z}_{2} \mid \mathbf{z}_{1}, \mathbf{z}_{2} \in \mathcal{C}_{i}, \mathbf{z}_{1} \neq \mathbf{z}_{2}\right\}$ be the set of difference vectors corresponding to the code book $\mathcal{C}_{i}$. Consider the set of matrices given by

$$
\Delta \mathcal{D}=\left\{\left[\mathbf{x}_{1}, \mathbf{x}_{2}, \ldots, \mathbf{x}_{n}\right] \mid \mathbf{x}_{1} \in \Delta \mathcal{C}_{1}, \mathbf{x}_{2} \in \Delta \mathcal{C}_{2}, \ldots, \mathbf{x}_{n} \in \Delta \mathcal{C}_{n}\right\}
$$

which is obtained by concatenating all possible difference vectors, one taken from each of the $n$ subsets. Note that each element in $\Delta \mathcal{D}$ is of size $\left(N_{t} \times n\right)$. Let $p=\min \{\operatorname{rank}(\mathbf{X}) \mid \mathbf{X} \in$ $\Delta \mathcal{D}\}$.

Proposition 1: The SM system employing EDAS achieves a diversity order of $N_{r} p$.

Proof: We adopt the steps in the proof of Theorem 2 in [20], showing that the rate of decay for the pairwise error probability (PEP) between any two TVs is at least $N_{r} p^{1}$.

Let the TVs in each codebook be indexed as $\mathcal{C}_{i}=\left\{\mathbf{x}_{l}(i) \mid l\right.$ $\left.\in\left\{1,2, N_{S M} M\right\}\right\}$. Let the optimal set of TAs for a given channel realization $\mathbf{H}$ be $I_{k^{*}}$ as in (2). Then the PEP between any two distinct TVs indexed by $l_{1}, l_{2}$ in the codebook $\mathcal{C}_{k^{*}}$ is given by

\footnotetext{
${ }^{1}$ The Proposition 1 in our paper is a generalization of Theorem 2 in [20] which is used along with Proposition 2 to arrive at our main result. Note that Proposition 2 constitutes the main result of our paper.
}

$$
\begin{aligned}
P E P\left(\mathbf{x}_{l_{1}} \rightarrow \mathbf{x}_{l_{2}} \mid \mathbf{H}\right) & =Q\left(\frac{\sqrt{\rho}}{2}\left\|\mathbf{H}\left(\mathbf{x}_{l_{1}}\left(k^{*}\right)-\mathbf{x}_{l_{2}}\left(k^{*}\right)\right)\right\|\right),(4) \\
& \leq \frac{1}{2} \exp \left(-\frac{\rho}{4}\left\|\mathbf{H}\left(\mathbf{x}_{l_{1}}\left(k^{*}\right)-\mathbf{x}_{l_{2}}\left(k^{*}\right)\right)\right\|(5)\right) .
\end{aligned}
$$

We now have to show that the $P E P$ decays as $1 / \rho^{N_{r} p}$. Let $\mathbf{x}_{\text {min }}(k)=\arg \min _{\mathbf{x} \in \Delta \mathcal{C}_{k}}\|\mathbf{H x}\|^{2}$ for $1 \leq k \leq n$ represent the difference vector from $\Delta \mathcal{C}_{k}$ corresponding to the minimum $\mathrm{ED}$ and $\mathbf{X}_{\min }=\left[\mathbf{x}_{\min }(1), \mathbf{x}_{\min }(2),, \mathbf{x}_{\min }(n)\right]$. Then we have

$$
\begin{aligned}
\left\|\mathbf{H}\left(\mathbf{x}_{l_{1}}\left(k^{*}\right)-\mathbf{x}_{l_{2}}\left(k^{*}\right)\right)\right\|^{2} & \geq\left\|\mathbf{H} \mathbf{x}_{\min }\left(k^{*}\right)\right\|^{2}, \\
& \geq \frac{1}{n}\left\|\mathbf{H} \mathbf{x}_{\min }\right\|_{F}^{2} .
\end{aligned}
$$

The inequality in (7) is due to the fact that $\mathbf{x}_{\min }\left(k^{*}\right)$ corresponds to the maximum ED among the elements in $\mathbf{X}_{m i n}$. Furthermore, we have

$$
\begin{aligned}
\left\|\mathbf{H} \mathbf{X}_{m i n}\right\|_{F}^{2} & =\operatorname{Tr}\left(\mathbf{X}_{m i n}^{H} \mathbf{H}^{H} \mathbf{H} \mathbf{X}_{m i n}\right), \\
& =\operatorname{Tr}\left(\mathbf{H} \mathbf{X}_{\min } \mathbf{X}_{m i n}^{H} \mathbf{H}^{H}\right), \\
& =\operatorname{Tr}\left(\overline{\mathbf{H}} \mathbf{D}_{\min } \overline{\mathbf{H}}^{H}\right),
\end{aligned}
$$

where $\mathbf{X}_{\min } \mathbf{X}_{\text {min }}^{H}=\mathbf{U} \mathbf{D}_{\min } \mathbf{U}^{H}$ is the Eigen decomposition of $\mathbf{X}_{\min } \mathbf{X}_{\min }^{H}$ and $\overline{\mathbf{H}}=\mathbf{H U}$. Since $\left\|\mathbf{H} \mathbf{X}_{m i n}\right\|_{F}^{2}=$ $\sum_{i=1}^{N_{r}} \sum_{j=1}^{q} \lambda_{j}\left|\bar{h}_{i, j}\right|^{2}$ where $q=\operatorname{rank}\left(\mathbf{X}_{\text {min }}\right) \geq p$, we have

$$
\left\|\mathbf{H} \mathbf{X}_{\text {min }}\right\|_{F}^{2} \geq \sum_{i=1}^{N_{r}} \sum_{j=1}^{p} \lambda_{j}\left|\bar{h}_{i, j}\right|^{2} \geq \lambda^{*} \sum_{i=1}^{N_{r}} \sum_{j=1}^{p}\left|\bar{h}_{i, j}\right|^{2},
$$

where $\lambda^{*}=\min _{\mathbf{X} \in \Delta D} \lambda_{s}\left(\mathbf{X X}^{H}\right)$. Thus, from (7) and (8) we arrive at:

$$
P E P\left(\mathbf{x}_{l_{1}} \rightarrow \mathbf{x}_{l_{2}} \mid \mathbf{H}\right) \leq \frac{1}{2} \exp \left(-\frac{\rho \lambda^{*}}{4 n} \sum_{i=1}^{N_{r}} \sum_{j=1}^{p}\left|\bar{h}_{i, j}\right|^{2}\right) .
$$

Therefore averaging over $\mathbf{H}$, we have

$$
P E P\left(\mathbf{x}_{l_{1}} \rightarrow \mathbf{x}_{l_{2}}\right) \leq \frac{1}{2} \prod_{i=1}^{N_{r}} \prod_{j=1}^{p} \mathrm{E}\left(\exp \left(-\frac{\rho}{4 n} \lambda^{*}\left|\bar{h}_{i, j}\right|^{2}\right)\right) .
$$

Since $\left|\bar{h}_{i, j}\right|^{2}$ is exponentially distributed with unit mean, we have

$$
\operatorname{PEP}\left(\mathbf{x}_{l_{1}} \rightarrow \mathbf{x}_{l_{2}}\right) \leq \frac{1}{2} \prod_{i=1}^{N_{r}} \prod_{j=1}^{p}\left(1+\frac{\rho \lambda^{*}}{4 n}\right)^{-1} .
$$

At a high SNR we have $\frac{\rho \lambda^{*}}{4 n} \gg 1$, hence

$$
P E P\left(\mathbf{x}_{l_{1}} \rightarrow \mathbf{x}_{l_{2}}\right) \leq \frac{1}{2}\left(\frac{\rho \lambda^{*}}{4 n}\right)^{-N_{r} p} .
$$

Thus, the probability of symbol errors in EDAS at high SNRs can be bounded as

$$
\begin{aligned}
P_{e} & \leq \frac{1}{\left|\mathcal{C}_{k^{*}}\right|} \sum_{\mathbf{x}_{l_{1}} \in \mathcal{C}_{k^{*}}} \sum_{\mathbf{x}_{l_{1}} \neq \mathbf{x}_{l_{2} \in \mathcal{C}_{k^{*}}}} \operatorname{PEP}\left(\mathbf{x}_{l_{1}} \rightarrow \mathbf{x}_{l_{2}}\right), \\
& \leq \frac{\left(\left|\mathcal{C}_{k^{*}}\right|-1\right)}{2}\left(\frac{\rho \lambda^{*}}{4 n}\right)^{-N_{r} p},
\end{aligned}
$$




$$
=\frac{\left(N_{S M} M-1\right)}{2}\left(\frac{\rho \lambda^{*}}{4 n}\right)^{-N_{r} p} .
$$

Note that the exponent of $\rho$ is $N_{r} p$. This concludes the proof.

Proposition 2: The SM system employing EDAS achieves a diversity order of $N_{r}\left(N_{t}-N_{S M}+1\right)$.

Proof: Owing to Proposition 1, it is sufficient to prove that we have $p \geq N_{t}-N_{S M}+1$ in order to prove the proposition. Let us now consider any matrix $\mathbf{X}$ from the set $\Delta \mathcal{D}$. We can then show that this matrix has at least $\left(N_{t}-N_{S M}+1\right)$ linearly independent columns and hence has a rank of at least $\left(N_{t}-\right.$ $\left.N_{S M}+1\right)$.

Let $\Delta S$ denote the set of non-zero difference constellation points given by $\left\{s_{1}-s_{2} \mid s_{1} \in S, s_{2} \in S, s_{1} \neq s_{2}\right\}$. Every element in $\Delta \mathcal{C}_{i}$ is either from the set $\mathcal{E}_{k}=$ $\left\{c \mathbf{e}_{k} \mid c \in \Delta S\right\}$ for $1 \leq k \leq N_{t}$, or from the set $\mathcal{E}_{p, q}=$ $\left\{c_{1} \mathbf{e}_{p}+c_{2} \mathbf{e}_{q} \mid c_{1}, c_{2} \in S, 1 \leq \bar{p} \neq q \leq N_{t}\right\}$. We show that it is impossible to construct a matrix $\mathbf{X}$ with rank less than $N_{t}-N_{S M}+1$ by picking columns from the set $\mathcal{E}_{k} \cup \mathcal{E}_{p, q}$.

First, we show that by restricting the columns of $\mathbf{X}$ to be from the collection of sets $\left\{\mathcal{E}_{k}\right\}$ the least possible rank of $\mathbf{X}$ can be $N_{t}-N_{S M}+1$. Then, we show that by picking the columns from the collection of sets $\left\{\mathcal{E}_{k} \cup \mathcal{E}_{p, q}\right\}$ the minimum achievable rank of $\mathbf{X}$ does not reduce than that achieved with $\mathcal{E}_{k}$ alone.

Pick $g_{1} \in \mathcal{E}_{k}$ for some legitimate $k$. Since $g_{1}$ belongs to $\left(\begin{array}{c}N_{t}-1 \\ N_{S M}-1\end{array}\right)$ of the $n$ sets $\left\{\Delta \mathcal{C}_{i}\right\}_{i=1}^{n}$, we pick $g_{1}$ from these $\Delta \mathcal{C}_{i}$ 's that constitute say first $\left(\begin{array}{c}N_{t}-1 \\ N_{S M}-1\end{array}\right)$ columns of $\mathbf{X}$. Similarly, we pick $g_{2} \in \mathcal{E}_{l}, l \neq k$. Since $g_{2}$ belongs to $\left(\begin{array}{c}N_{t}-2 \\ N_{S M}-1\end{array}\right)$ of the remaining $\left(\begin{array}{c}n-N_{t}-1 \\ N_{S M}-1\end{array}\right)$ sets in the collection $\left\{\Delta \mathcal{C}_{i}\right\}$, we pick $g_{2}$ from these sets that constitute the next $\left(\begin{array}{c}N_{t}-2 \\ N_{S M}-1\end{array}\right)$ columns of $\mathbf{X}$. Now we have picked $\left(\begin{array}{c}N_{t}-1 \\ N_{S M}-1\end{array}\right)+\left(\begin{array}{c}N_{t}-2 \\ N_{S M}-1\end{array}\right)$ columns of $\mathbf{X}$, which has a rank equal to two. Proceeding in the same lines, at the $m^{t h}$ step, we would have chosen $\sum_{i=1}^{m}\left(\begin{array}{c}N_{t}-i \\ N_{S M}-1\end{array}\right)$ columns of $\mathbf{X}$, which has a rank equal to $m$. Since $n=\sum_{i=1}^{N_{t}-N_{S M}+1}\left(\begin{array}{c}N_{t}-i \\ N_{S M}-1\end{array}\right)$, we would have picked all the columns of $\mathbf{X}$ when $m=N_{t}-N_{S M}+1$. Thus the minimum rank of $\mathbf{X}$ when the columns are picked from the collection $\left\{\mathcal{E}_{k}\right\}$ is $N_{t}-N_{S M}+1$. Now we show that by considering the set $\left\{\mathcal{E}_{k} \cup \mathcal{E}_{p, q}\right\}$, we will not have any advantage in reducing the rank of $\mathbf{X}$ any further.

Suppose, assume that we pick $g_{1}^{\prime} \in \operatorname{span}\left(\mathbf{e}_{l}, \mathbf{e}_{m}\right)$ from the set $\mathcal{E}_{p, q}$ instead of $g_{1} \in \mathcal{E}_{k}$. Since $g_{1}^{\prime}$ belongs to $\left(\begin{array}{c}N_{t}-2 \\ N_{S M}-2\end{array}\right)$ of the $n$ sets, which is strictly lesser ${ }^{2}$ than $\left(\begin{array}{c}N_{t}-1 \\ N_{S M}-1\end{array}\right)$, any choice of the vector from the collection $\left\{\mathcal{E}_{k} \bigcup \mathcal{E}_{p, q}\right\}$ to fill in the remaining $\left(\begin{array}{c}N_{t}-1 \\ N_{S M}-1\end{array}\right)-\left(\begin{array}{c}N_{t}-2 \\ N_{S M}-2\end{array}\right)$ columns will

$$
\begin{aligned}
& { }^{2} \text { Note that }\left(\begin{array}{c}
N_{t}-1 \\
N_{S M}-1
\end{array}\right)=\left(\begin{array}{c}
N_{t}-2 \\
N_{S M}-1
\end{array}\right)+\left(\begin{array}{c}
N_{t}-2 \\
N_{S M}-2
\end{array}\right) \text {, and hence } \\
& \left(\begin{array}{c}
N_{t}-2 \\
N_{S M}-2
\end{array}\right)<\left(\begin{array}{c}
N_{t}-1 \\
N_{S M}-1
\end{array}\right) .
\end{aligned}
$$

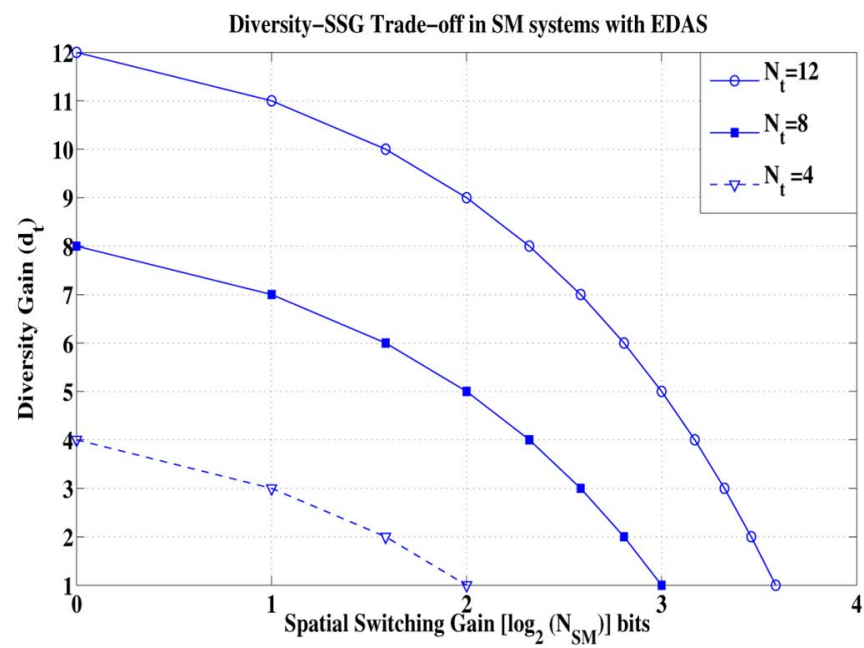

Fig. 1. Trade-off between transmit diversity and switching gain in SM systems employing EDAS with various number of transmit antennas.

increase the rank of $\mathbf{X}$ to two, whereas $g_{1}$ gives a rank of only one for $\left(\begin{array}{c}N_{t}-1 \\ N_{S M}-1\end{array}\right)$ columns. However, we can pick vectors whose basis element is either $\mathbf{e}_{l}$ or $\mathbf{e}_{m}$ while increasing the rank of $\mathbf{X}$ only by one. There are totally $\left(\begin{array}{c}N_{t}-1 \\ N_{S M}-1\end{array}\right)$ vectors for each of the basis. Since we have already chosen those columns which have both $\mathbf{e}_{l}$ and $\mathbf{e}_{m}$, we have effectively $\left(\begin{array}{c}N_{t}-1 \\ N_{S M}-1\end{array}\right)+\left(\begin{array}{c}N_{t}-1 \\ N_{S M}-1\end{array}\right)-\left(\begin{array}{c}N_{t}-2 \\ N_{S M}-2\end{array}\right)$ columns, which is equal to $\left(\begin{array}{c}N_{t}-1 \\ N_{S M}-1\end{array}\right)+\left(\begin{array}{c}N_{t}-2 \\ N_{S M}-1\end{array}\right)$. Now, $\mathbf{X}$ has a rank of two with $\left(\begin{array}{c}N_{t}-1 \\ N_{S M}-1\end{array}\right)+\left(\begin{array}{c}N_{t}-2 \\ N_{S M}-1\end{array}\right)$ columns filled. Note that by restricting the columns of $\mathbf{X}$ to be from $\mathcal{E}_{k}$, a rank of two was achieved with the same number of columns filled. Thus, picking elements from $E_{p, q}$ will not give any advantage in reducing the rank further. This concludes the proof.

Thus, it becomes clear from Proposition 2 that the SM system cannot achieve full transmit diversity, while achieving a nonzero SSG. Fig. 1 compares the trade-off between the achievable transmit diversity and SSG for various number of TAs with different choices of $N_{S M}$. Considering for example $N_{t}=12$, we can have $N_{S M}=[2,4,8] \leq N_{t}$. The achievable transmit diversity gains in these cases are 11,9 , and 5 respectively. Note that $N_{S M}=1$ corresponds to the conventional TA selection scheme of [17].

\section{Simulation Results AND Discussion}

Simulation Scenario: In all our simulations, we assume block Rayleigh fading channels and perfect channel estimation at the receiver. Furthermore, the receiver is assumed to perform maximum-likelihood detection in all the transmission schemes considered. For each channel realization, the antenna subset $I_{E D}$ of (2) is obtained by employing the low-complexity EDAS algorithm given in [16].

We demonstrate that the SM system employing EDAS indeed achieves a transmit diversity of order $d_{t}=\left(N_{t}-N_{S M}+1\right)$. Consider an SM system operating with $N_{t}=5, N_{r}=1$, 16-QAM and 32-QAM signal sets, where $N_{S M} \in[1,2,3,4]$. The choice of $N_{r}=1$ ensures that the achieved diversity is only 


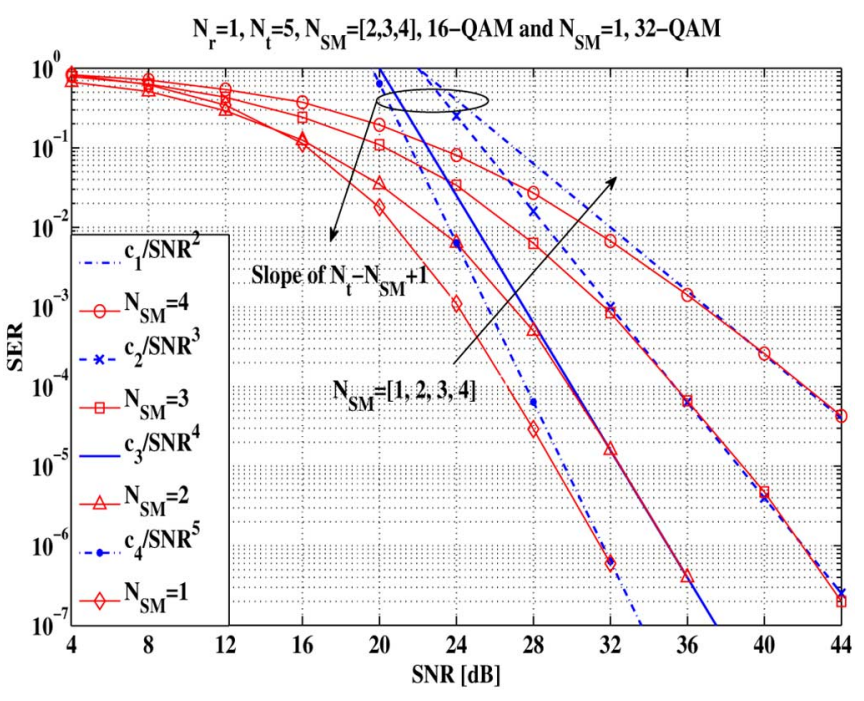

Fig. 2. SER performance of the SM system having $N_{r}=1, N_{t}=5$ and employing 16-QAM signal set for $N_{S M} \in[2,3,4]$.

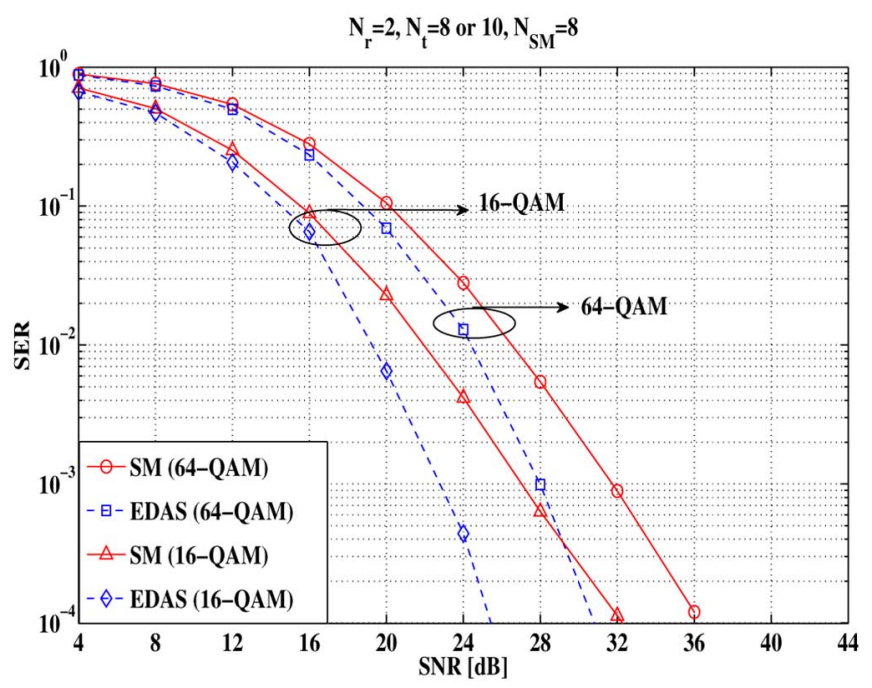

Fig. 3. SER performance of the SM system having $N_{r}=2, N_{t}=10$ or 8 , $N_{S M}=8$ and employing 16- and 64-QAM signal sets.

due to TA subset selection. Fig. 2 gives the SER performance of the above SM system for $N_{S M} \in[1,2,3,4]$ along with the reference curves of the form $c / S N R^{N_{t}-N_{S M}+1}$, where $c$ is an appropriately chosen positive constant ${ }^{3}$. It is clear from Fig. 2 that the SM system employing EDAS achieves a transmit diversity order of $\left(N_{t}-N_{S M}+1\right)$.

Consider an SM system having $N_{r}=2, N_{t}=10$ and employing EDAS using $N_{S M}=8$. Also consider an SM system having $N_{r}=2$ and $N_{t}=8$. Fig. 3 provides the SER comparison of these systems when using 16- and 64-QAM signal sets. It is evident from Fig. 3 that EDAS increases the diversity order. Specifically, at an SER of about $10^{-4}$ the SNR gain achieved by

\footnotetext{
${ }^{3}$ The constants chosen in Fig. 2 are $c_{1}=2.515 \times 10^{4}, c_{2}=4 \times 10^{6}$, $c_{3}=10^{8}$ and $c_{4}=64 \times 10^{8}$.
}

EDAS is about $5 \mathrm{~dB}$ in case of $64-\mathrm{QAM}$ and about $7 \mathrm{~dB}$ in case of 16-QAM.

\section{CONCLUSIONS}

In this paper, we have quantified the achievable transmit diversity order of SM employing EDAS. Furthermore, the resultant trade-off between the diversity order and the switching gain was quantified. Our simulation results confirmed the theoretical formulae.

\section{REFERENCES}

[1] R. Mesleh, H. Haas, C. Ahn, and S. Yun, "Spatial modulation - a new low complexity spectral efficiency enhancing technique," in Proc. 2006 First Int. Conf. Commun. Netw., Beijing, China, pp. 1-5.

[2] R. Mesleh, H. Haas, S. Sinanovic, C. Ahn, and S. Yun, "Spatial modulation," IEEE Trans. Veh. Technol., vol. 57, no. 4, pp. 2228-2242, 2008

[3] M. Di Renzo and H. Haas, "Performance comparison of different spatial modulation schemes in correlated fading channels," in Proc. 2010 IEEE Int. Conf. Commun. (ICC), Cape Town, South Africa.

[4] E. Basar, U. Aygolu, E. Panayirci, and H. V. Poor, "New trellis code design for spatial modulation," IEEE Trans. Wireless Commun., vol. 10, no. 9, pp. 2670-2680, Aug. 2011.

[5] E. Basar, U. Aygolu, E. Panayirci, and H. V. Poor, "Performance of spatial modulation in the presence of channel estimation errors," IEEE Commun. Lett., vol. 16, no. 2, pp. 176-179, Feb. 2012.

[6] J. Zheng, "Signal vector based list detection for spatial modulation," IEEE Wireless Commun. Lett., vol. 1, no. 4, pp. 265-267, Aug. 2012.

[7] Q. Tang, Y. Xiao, P. Yang, Q. Yu, and S. Li, "A new low-complexity near-ML detection algorithm for spatial modulation," IEEE Wireless Commun. Lett., vol. 2, no. 1, pp. 90-93, Feb. 2013.

[8] R. Mesleh and S. S. Ikki, "Performance analysis of spatial modulation with multiple decode and forward relays," IEEE Wireless Commun. Lett., vol. 2, no. 4, pp. 423-426, Aug. 2013.

[9] S. Sugiura and L. Hanzo, "Effects of channel estimation on spatial modulation," in IEEE Signal. Process. Lett., Dec. 2012, vol. 19, no. 12 , pp. $805-808$.

[10] M. Di Renzo and H. Haas, "Space shift keying (SSK) modulation: On the transmit-diversity/multiplexing trade-off," in Proc. 2011 IEEE Int. Conf. Commun. (ICC), Kyoto, Japan.

[11] S. Sugiura, S. Chen, and L. Hanzo, "Coherent and differential space-time shift keying: A dispersion matrix approach," IEEE Trans. Commun., vol. 58, no. 11, pp. 3219-3230, 2010.

[12] M. Di Renzo and H. Haas, "Transmit-diversity for spatial modulation (SM): Towards the design of high-rate spatially-modulated space-time block codes," in Proc. 2011IEEE Int. Conf. Commun. (ICC), Kyoto, Japan.

[13] E. Basar, U. Aygolu, E. Panayirci, and H. V. Poor, "Space-time block coding for spatial modulation," IEEE Trans. Commun., vol. 59, pp. 823-832, Mar. 2011.

[14] P. Yang, Y. Xiao, Y. Yu, and S. Li, "Adaptive spatial modulation for wireless MIMO transmission systems," IEEE Commun. Lett., vol. 15, no. 6, pp. 602-604, Jun. 2011.

[15] P. Yang, Y. Xiao, L. Li, Q. Tang, Y. Yu, and S. Li, "Link adaptation for spatial modulation with limited feedback," IEEE Trans. Veh. Technol., vol. 61, no. 8, pp. 3808-3813, Oct. 2012.

[16] R. Rajashekar, K. V. S. Hari, and L. Hanzo, "Antenna selection in spatial modulation systems," IEEE Commun. Lett., vol. 17, no. 3, pp. 521-524, Mar. 2013.

[17] S. Sanayei and A. Nosratinia, "Antenna selection in MIMO systems," IEEE Commun. Mag., vol. 42, no. 10, pp. 68-73, Oct. 2004.

[18] D. Love, R. Heath, V. Lau, D. Gesbert, B. Rao, and M. Andrews, "An overview of limited feedback in wireless communication systems," IEEE J. Sel. Areas Commun., vol. 26, no. 8, pp. 1341-1365, Oct. 2008.

[19] R. Heath and A. Paulraj, "Antenna selection for spatial multiplexing systems based on minimum error rate," in Proc. 2001 Int. l Conf. Commun., Helsinki, Finland, vol. 7, pp. 2276-2280.

[20] L. P. Natarajan and B. S. Rajan, "Full-rate, full-diversity, finite feedback space-time schemes with minimum feedback and transmission duration," IEEE Trans. Wireless Commun., vol. 12, no. 10, pp. 5022-5034, Oct. 2013. 